\title{
Optical Design of a Dual Channel Microscope
}

\author{
Anastasiya Kozhina ${ }^{1[0000-0003-3547-9771]}$, Evgeniia Soshnicova ${ }^{10000-0003-4847-5825]}$ and Alla \\ Uvarova ${ }^{1[0000-0002-5075-8376]}$ \\ ${ }^{1}$ ITMO University, St. Petersburg, Russia \\ crensta@mail.ru
}

\begin{abstract}
The article presents a new scheme of an optical microscope. The proposed scheme contains two channels: one works with a wide field of view, the other forms a high-resolution image. The simultaneous fulfillment of these conditions significantly increases the information capacity of the system. The proposed scheme overcomes the limitations imposed by the Lagrange invariant. In addition, the presented work solves the relevant task of optical instrumentation: using one device, it becomes possible to simultaneously obtain an image of an object in two scales, one of which gives a detailed image of microstructures, and the other shows the state of the area around the part of the object being studied in detail. This time synchronization is especially important for studying biological objects. The design features of the optical scheme make it possible to abandon the refocusing of the objective, which is necessary in biological microscopes when the magnification is changed. Thus, the proposed system solves not only scientific and technical tasks, but also improves the ergonomics of work in microscopy.
\end{abstract}

Keywords: Microscope, Optical design, Light microscopy.

\section{Introduction}

Microscope is one of traditional types of optical devices used to research of microstructures. Working with a microscope is closely related to the peculiarities of how operator perceives the object under research. It is known that before the formation of a sensory image in a person's perception, there is a detection, distinction and identification of an object (in whole or in its details). It should be noted that systems of machine vision try to implement a similar algorithm of working with objects [1, 2]. In the case of observation with unaided eye, all stages of shaped vision (from isolating the object of research from the general background to identifying its main features) occur sequentially [3] with the help of eye accommodation and refocusing of operator's attention.

The purpose of using optical devices is empowerment of human eye. Each of the mentioned stages of perception assume the discrimination of smaller elements; there-

Copyright (C) 2020 for this paper by its authors. Use permitted under Creative Commons License Attribution 4.0 International (CC BY 4.0). 
fore, the optical device should help to increase the resolving power of the operator's eye. In visual instruments, this is associated with an increase in magnification. The traditional solution of this purpose in microscopy is using of a revolver with several micro-objectives in the device.

Nowadays, high requirements on optical devices in terms of the quality of the resulting image are imposed. Also, consumers often want to observe as many objects as possible at the same time. However, the ability of an optical system to provide a highresolution image in a wide field is impossible on the basis of the Lagrange invariant [4], which expresses the law of conservation of information in geometric optics. The Lagrange invariant connects the aperture and size of the object with the aperture and size of the image, thereby imposing a restriction on the transformation of beams.

Changing the magnifications in the microscope takes away a significant part of the information about the object: the operator is deprived of the opportunity to observe what is locating around the research field, which is always subject of great interest in the case of living biological samples.

In addition, the change in magnifications disrupts the continuity of the observation process, therefore, does not allow use operator's psychophysical abilities effectively. Also, this action takes time to refocus the system, which can be critical when studying samples that change their appearance over time: they can be lost from the field of view or, in the case of living cells that are sensitive to light, irradiate them in vain, wasting their life resource only to configure the system [5].

As a result, there is a contradiction: the operator is either limited in resolution but retains the possibility of indirect observation of the rest of the field, or acquires the ability to see details with high resolution, but only in a very small area. Average magnification with an average field of view is a compromise solution that includes the disadvantages of both options rather than their advantages.

The purpose of this work is to design an optical scheme of a microscope that allows observing objects simultaneously in a wide field and with a high resolution. The use of such systems will make it possible to eliminate the need of lens refocusing arising from a sharp decrease in the depth of field and will also provide the possibility of detecting and identifying the structures under research without reconfiguring of visual instrument.

\section{Modern Microscopes}

As stated in the Abbe diffraction theory, the resolution of a microscope is directly related to its numerical aperture and wavelength [6].

$$
\sigma=\frac{\lambda}{2 N A}
$$

In the case of work in the visible range, the resolution of the microscope entirely depends on its numerical aperture. However, as mentioned above, observation of an image at once in a wide field and with a high aperture is limited according to the Lagrange invariant. 
In order to overcome this restriction, it was decided to develop a dual-channel optical system. Such systems are widely used in optical instrumentation and in microscopy in particular. Examples of instruments with observation in two channels are comparative microscopes and forensic microscopes. For instance, the LEICA FS CB [7] microscope shown in Figure 1. Microscopes of this type have two channels and are designed to compare two objects. Although such instruments have the theoretical possibility of observing objects in one channel with a wide field of view and in the other with a high aperture, using these types of microscopes experts research two different objects.

An example of two-channel devices, both channels of which are directed to the same object, are microscopes using interference methods. They have found wide application in optical coherence tomography [8-9]. However, only one of these channels is for object observation. Another channel of these microscopes is for illumination.

Wide-field high-aperture microscopes are also known [10-11]. For example, electronic and fluorescence microscopes. Nevertheless, they use methods other than optical ones, therefore they cannot be analogues of this development.

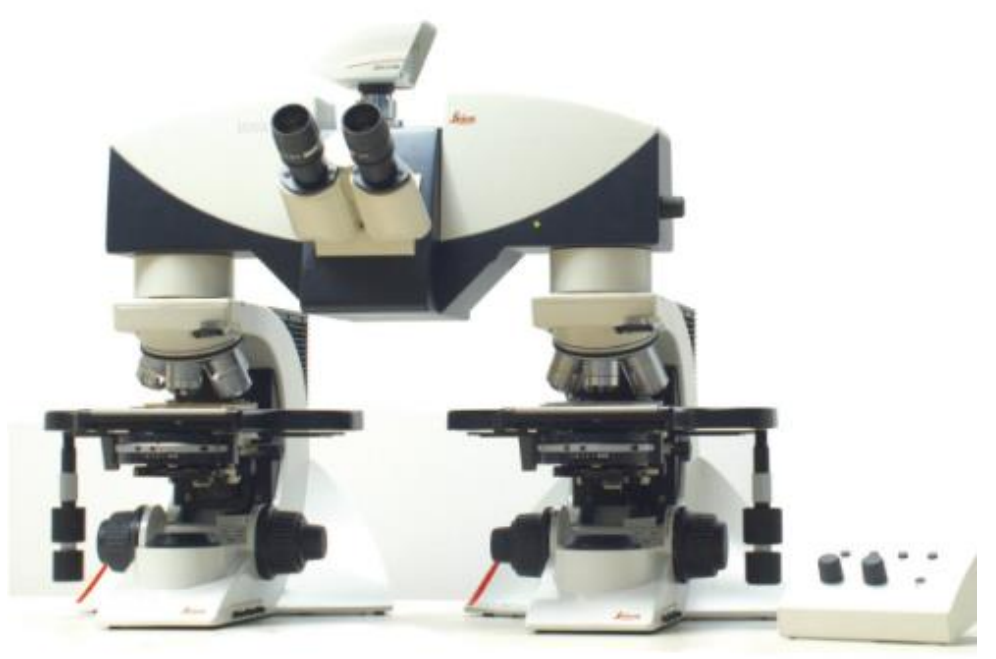

Fig. 1.Comparative mechanical microscope for the research of micro-objects LEICA FS CB

\section{Prototype of the Development Device}

The prototype of the development is basic optical design of the research metallographic microscope MIM-7 [12] (see Fig. 2). It is classified as light microscope and its frontal objective 12 provides two-channel operation.

The illumination channel includes a light source 1 , the rays from which are directed by the collector 2 to the mirror 3 . Reflecting from the mirror, the light beam sequentially encounters on its way a light filter 4, aperture diaphragm 5, lens 6 (and 
polarizer 21, if it is included into system), a photo shutter 7 and a field diaphragm 8 . The pentaprism 9 re-reflects the beam, which is then refracted by the lens 10 and falls on the semitransparent reflective plate 11 .

The scheme of the lighting channel is completed by microlens 12 and illuminated research subject 13. At the same time, at this place the observation channel begins: in a reverse beam path from sample through the microlens 12 and the beam-splitting semitransparent plate 11. After the beam-splitting plate 11 the light passes through the plug-in analyzer 20 and is refracted by achromatic lens 14. Depending on the selected method of observation (visually or with a photodetector) operator can change the position of the mirror 18 . When the mirror 18 is included into the system, the beam of rays enters the eyepiece. To photograph the object, the mirror is removed from the system, and the beam of rays continues to move through the photo eyepiece 15 , is reflected by the mirror 16 and falls on the image receiver 17 .

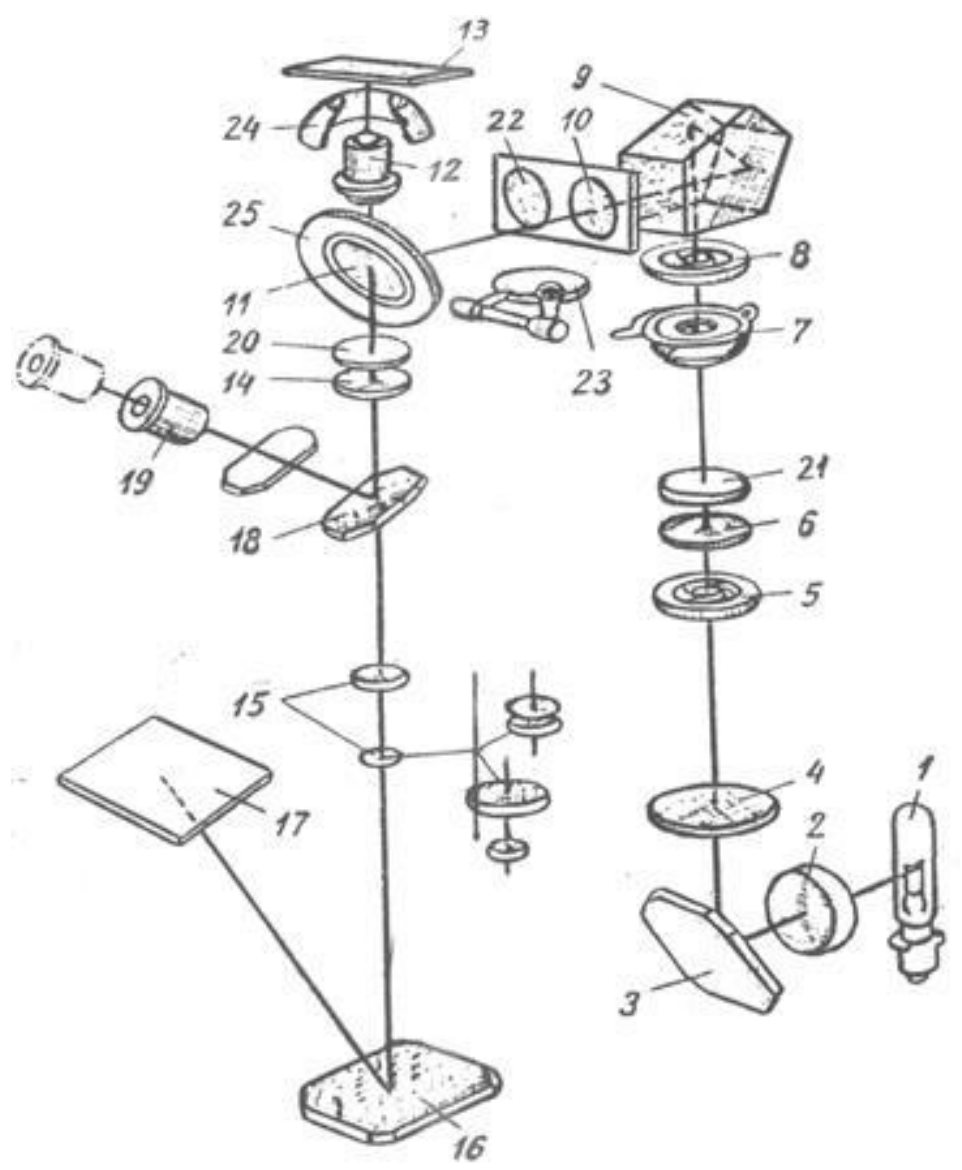

Fig. 2. Optical system of the metallographic microscope MIM-7 
As mentioned above, the lens 12 provides two channels. It is designed as objective with infinity tube, which allows the division of light without adding aberrations. However, such a lens is not designed for use with a cover glass that is necessary for the research of biological objects, which, at high apertures, will add large spherical aberration. Furthermore, there is only one observation channel in the optical scheme of a metallographic microscope. The illumination in this system is in reflected light, while the research of biomaterial requires illumination in transmitted light. Based on the foregoing, although the optical scheme of metallographic microscope MIM-7 is the basis for the optical system under development, its basic optical design is far from being a complete analogue.

\section{$4 \quad$ Layout Description}

The proposed scheme of a dual-channel microscope is shown on Figure 3 and consists of a frontal, wide-field and high-aperture objectives, a beam splitter and optical image receivers.

The front lens is designed for simultaneous wide field and high aperture operation. Its presence removes the need for refocusing on the subject, which usually occurs with changing the magnification in laboratory microscopes.

A beam splitter separates the light into two channels. To minimize aberrations, it should have an aperture diaphragm. Moreover, a parallel beam of rays should fall on the beam splitter, since in case an oblique beam falls on the beam splitter, intractable coma and astigmatism will appear in the system [13-14]. For this reason, the front lens must be designed as objective with infinity tube. Moreover, it must be designed to work with a cover glass, which is necessary for the research of biological objects.

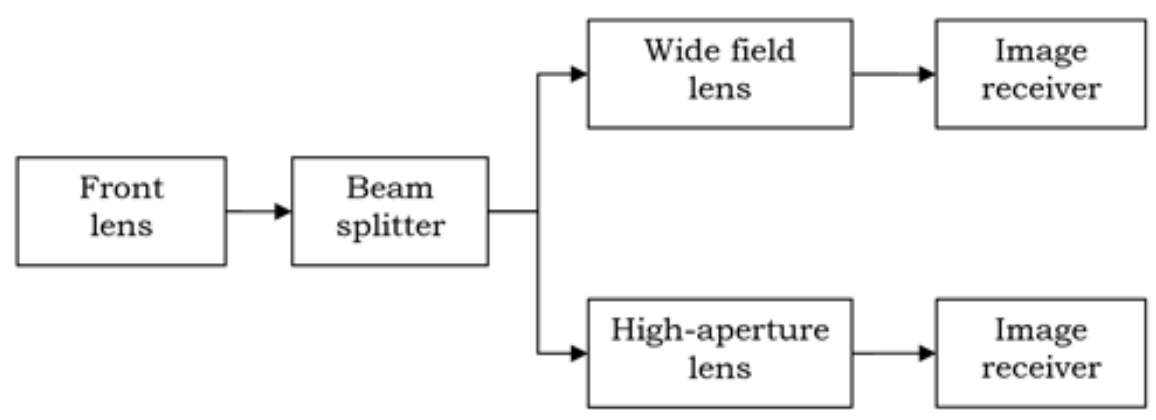

Fig. 3. Block diagram of a dual-channel microscope

The first channel will transmit information with a wide field, which is necessary for observing large objects, as well as detecting anomalies in biomaterial. A wide-field lens should provide the necessary correction of field aberrations: astigmatism, distor- 
tion, curvature. The second channel transmits information with high resolution, which allows a detailed study of the object of research. In this channel, it will be necessary to correct aberrations of wide beams of rays: coma, spherical aberration, spherochromatism.

Figure 4 shows the layout of dual-channel optical microscope in thin components. The wide-field channel magnification is $4 \mathrm{x}$, which corresponds to a number of standard microscope magnifications. The numerical aperture in this channel is 0.1. The second channel is designed for 40x magnification with an aperture of 0.5 . Working at this aperture will allow viewing objects with a size of $0.55 \mu \mathrm{m}$, according to (1).

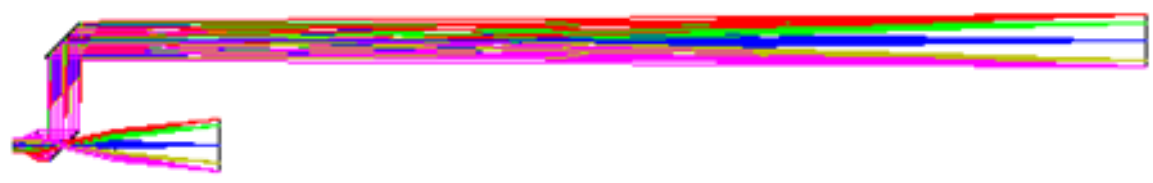

Fig. 4. Layout of dual-channel optical microscope

It is proposed to implement illumination in a dual-channel microscope according to Koehler (see Fig. 5) [15]. This lighting system consists of collector, condenser and two iris diaphragms: field and aperture. Source 1 is shown by collector 2 in the plane of aperture diaphragm 3 located in front focal plane of the condenser 4 . The field diaphragm 7 is projected by the condenser in the plane of the studied biological object 5. Also, condenser projects the aperture diaphragm into the entrance pupil of the objective 6, that is, at infinity. In dual-channel microscope it is necessary to highlight whole size of the object in the broadband channel with diaphragm 3 , and the numerical aperture of the high-aperture channel with diaphragm 7.

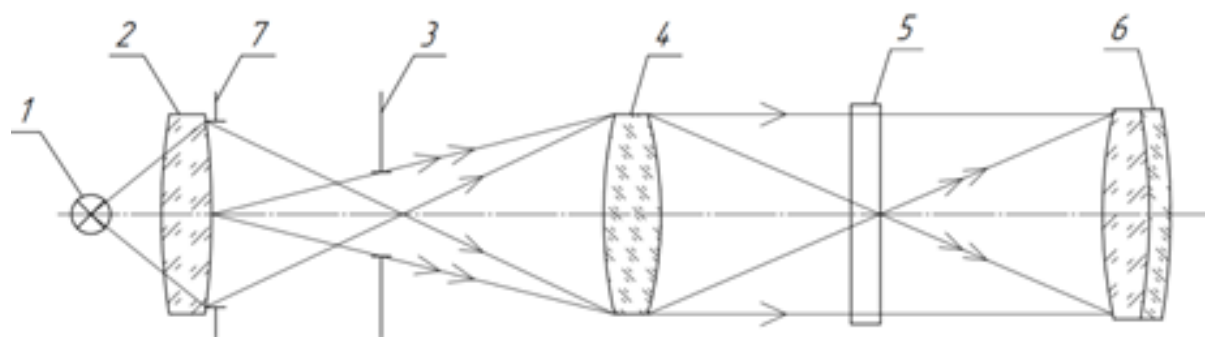

Fig. 5. Lighting layout according to Koehler

\section{Conclusion}

Modern analysis of biological materials requires increasing of the capabilities existing research instruments. The device based on the proposed scheme enables the operator to receive and register information about the object simultaneously on two scales. 
This demonstrates a new approach to solving the conflict between resolution rage of the device and its field of view. Also proposed instrument significantly improves the ergonomics of working with the microscope, eliminating the need for the operator to configure the device too many times.

\section{References}

1. Liu, B., Ju, H., Yao, Y. Object recognition and centroid detection based on machine vision. Second International Conference on Mechanic Automation and Control Engineering, (2011).

2. Chen, L., Singh, S., Kailath, T., Roychowdhury, V.:Brain-inspired automated visual object discovery and detection. Proceedings of the National Academy of Sciences, 116 (1), 96105 (2019).

3. Straube, S., Fahle, M.: Visual detection and identification are not the same: evidence from psychophysics and fMRI. Brain Cogn, 75(1), 29-38 (2011).

4. Greivenkamp, J.:Field Guide to Geometrical Optics. SPIE Publications, Washington (2004)

5. Swedlow, J., Platani, M.: Live cell imaging using wide-field microscopy and deconvolution. Cell Structure and Function, 27(5), 335-41 (2002).

6. Rottenfusser, R., Wilson, E., Davidson, M.: Numerical Aperture and Resolution,https://www.zeiss.com/microscopy/int/solutions/reference/basicmicroscopy/numerical-aperture-and-resolution.html,last accessed 2020/09/14.

7. Motorized Forensic Comparison Microscope Leica FS CB, https://www.leicamicrosystems.com/products/light-microscopes/p/leica-fs-cb/,last accessed 2020/09/14.

8. Gurov, I., Margaryants, N., Pimenov, A.:Line-field swept source optical coherence tomography system for evaluating microstructure of objects in near-infrared spectral range. Proc. SPIE 10333, Optical Methods for Inspection, Characterization, and Imaging of Biomaterials III (2017).

9. Shen, L., Carrasco-Zevallos, O., Keller, B., Viehland, C., Waterman, G.,Desouza, P., Hahn, P.,Kuo, A., Toth, C., Izatt, J.: Novel microscope-integrated stereoscopic display for intrasurgical optical coherence tomography.Biomedical Optics Express 7(5) (2016).

10. Shimomura, S., Nishimurab, T., Oguraa, Y., Tanidaa, J.: Reversible transformation of DNA gels using light signals, OPTICS and PHOTONICS International Congress 2019, pp. 105-107 (2019).

11. Forcucci, A., Pawlowski, M., Crannell, Z., Pavlova, I., Richards-Kortum, R., Tkaczyk, T.: All-plastic miniature fluorescence microscope for point-of-care readout of bead-based bioassays, Journal of Biomedical Optics 20(10) (2015).

12. Malyar, I., Stetsyura, S., Sinev, I.,Bratashov, D.: Micro- and macrostructure of multicomponent materials and structures based on them.Saratov (2014).

13. Grammatin, A., Chan, K.: Spectrum splitting optical system for devices operating simultaneously in two different areas of the IR spectrum. Scientific and technical bulletin of information technologies, mechanics and optics 43 (2007).

14. Chan, K.: Development and research of lens objectives for thermal imaging devices. Dissertation abstract. $\mathrm{SPb}(2008)$.

15. Davidson, M.: Köhler Illumination. https://www.zeiss.com/microscopy/int/solutions/reference/basic-microscopy/koehlerillumination.html, last accessed 2020/09/14. 